Hypertonie

47-Witwenhochdruck

51 - Therapiekontrolle

Prof. Dr. med. W. Zidek

Med. Klinik IV, Charité - Universitätsmedizin Berlin

\title{
Diagnostik und Therapie des Bluthochdrucks
}

\section{„Den Hypertoniker" gibt es nicht}

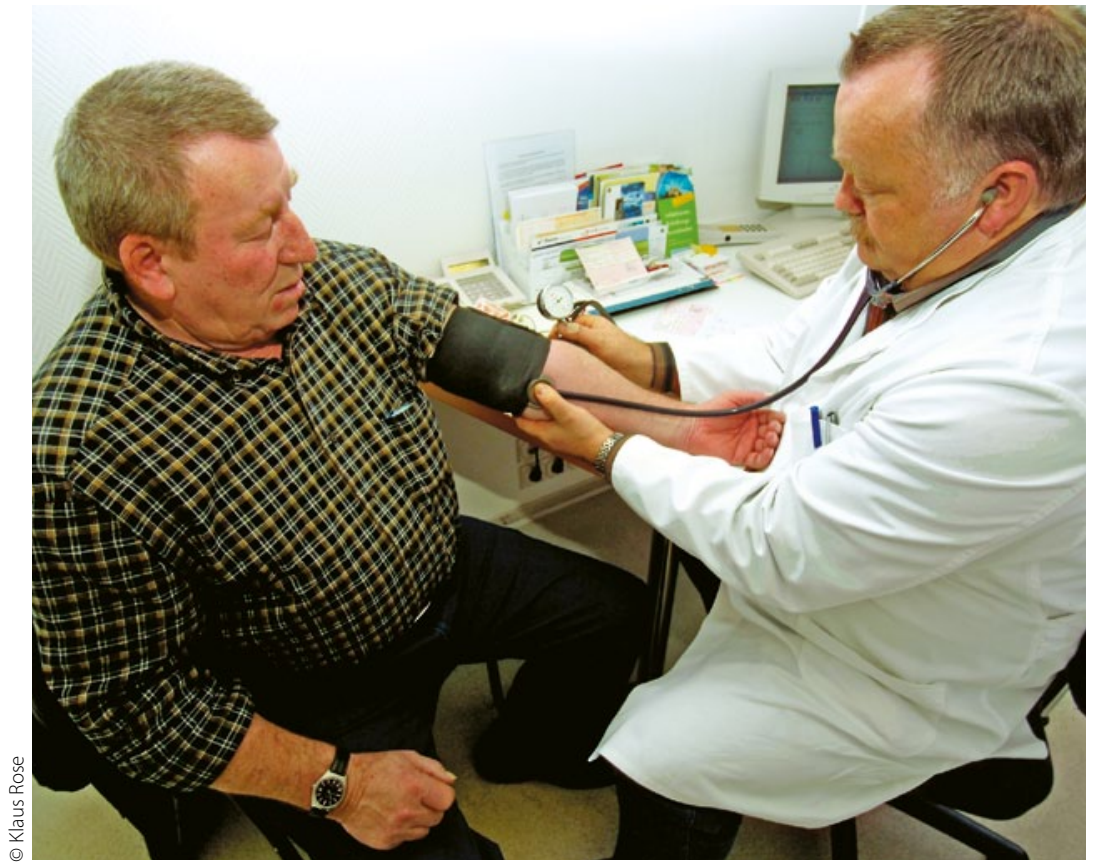

Bei Hochdruckpatienten nicht nur auf den Blutdruckwert achten!

Der Beitrag zur Therapiekontrolle fasst die wesentlichen Änderungen in der Hypertoniebehandlung zusammen. Weiterhin im Fluss dürfte die Frage der dualen Blockade des Renin-Angiotensin-Aldosteron-Systems sein. Einigkeit herrscht sicher darüber, dass die duale Blockade keine Routinetherapie für den essenziellen Hypertoniker ohne spezielle Probleme ist. Gegenwärtig dürfte der Favorit für den Behandlungsbeginn mit einer Kombination das Duo RAS-Blocker + Kalziumantagonist sein. Die bereits in der Fachliteratur häufig diskutierten Probleme mit dem Renininhibitor Aliskiren in der Kombination mit anderen RAS-Blockern zur Behandlung der diabetischen Nephropathie dürfte den Indikationsspielraum dieser Substanz wesentlich einengen.

Eine wichtige Änderung in der Hypertoniebehandlung sind die invasiven Verfahren, die renale Denervation und die Barorezeptor-Stimulation. Aus meiner Sicht ist für die Anwendung dieser Verfahren nach wie vor eine kritische Indikationsstellung wichtig. Maßstab für die Durchführung darf nicht die bloße Machbarkeit im technischen

— Die Hypertoniebehandlung wird zunehmend individueller. Wir erkennen immer mehr, dass es "den Hypertoniker" nicht gibt. Auch in den gängigen Therapieempfehlungen differenzieren wir immer stärker und finden, dass der Hypertoniepatient mit Diabetes spezielle Überlegungen erfordert, ebenso wie auch der Hypertoniker im höheren Lebensalter. Wie der Beitrag von M. Middeke zeigt, haben jetzt auch die Witwen den Status einer speziellen Gruppe von Hypertonikern erlangt. Natürlich ist der Begriff Witwenhochdruck nicht wörtlich zu nehmen. Er bezieht sich aber auf eine Gruppe von Patienten, die mit dem vielbeschworenen demografischen Wandel häufiger werden dürfte. Aufmerksame Beobachter werden feststellen, dass in ihrer Sprechstunde eine Gruppe von Patienten existiert, auf die die Merkmale des ,"Witwenhochdrucks" im Großen und Ganzen zutreffen.
Sinn sein. Eine kritische Indikationsstellung nach Abklärung sekundärer Hypertonieursachen sowie der häufigen Compliance-Probleme sowie die kritische Bewertung der eingesetzten Medikamente und ihrer Dosierung ist für den langfristigen Erfolg dieser Verfahren unverzichtbar.

Dieser Beitrag ist, neben den Hinweisen auf Änderungen in den Empfehlungen zur Hypertoniebehandlung, ein schönes Beispiel dafür, dass die Hypertoniebehandlung ständig neue Herausforderungen zu meistern hat. In einer Phase, in der die medikamentöse Therapie keine vielfältigen Neuerungen zu verzeichnen hat, wandeln sich unsere Hypertoniepatienten und ihre spezifischen therapeutischen Anforderungen. Ich wünsche den Lesern des Themenschwerpunkts Hypertonie viel Vergnügen und Anregungen für die Praxis der Hypertoniebehandlung. 